\title{
Efficacy, Practicality, and Safety of Inhaled Methoxyflurane in Elderly Patients with Acute Trauma Pain: Subgroup Analysis of a Randomized, Controlled, Multicenter, Open-Label Trial (MEDITA)
}

This article was published in the following Dove Press journal:

Journal of Pain Research

Sossio Serra'
Antonio Voza (D)
Germana Ruggiano
Andrea Fabbri iD
Elisabetta Bonafede ${ }^{5}$
Antonella Sblendido ${ }^{6}$
Amedeo Soldi (D)
Alberto Farina (DD

On behalf of the MEDITA
Study Group
'Emergency Department, Maurizio
Bufalini Hospital, Cesena, Italy;
${ }^{2}$ Emergency Department, IRCCS
Humanitas Research Teaching Hospital,
Rozzano, Milan, Italy; ${ }^{3}$ Emergency
Medicine Department, Santa Maria
Annunziata Hospital, Florence, Italy;
${ }^{4}$ Department of Emergency Medicine,
Morgagni-Pierantoni Hospital, Forli, Italy;
${ }^{5}$ YGHEA, CRO Division of Ecol Studio
Bioikos S.r.l., Bologna, Italy; ${ }^{6}$ Medical
Affairs Department, Mundipharma
Pharmaceuticals Srl, Milan, Italy

Sossio Serra'

Antonio Voza (iD $)^{2}$

Germana Ruggiano ${ }^{3}$

Andrea Fabbri (D)

Elisabetta Bonafede (D)

Antonella Sblendido ${ }^{6}$

Amedeo Soldi $\mathbb{D}^{6}$

Alberto Farina $\mathbb{1 D}^{6}$

On behalf of the MEDITA

\begin{abstract}
${ }^{2}$ Emergency Department,
Humanitas Research Teaching Hospital,

Rozzano, Milan, Italy; ${ }^{3}$ Emergency

Annunziata Hospital, Florence, Italy;

${ }^{4}$ Department of Emergency Medicine,

Morgagni-Pierantoni Hospital, Forli, Italy;

Bioikos S.r.l., Bologna, Italy; ${ }^{6}$ Medical

Pharmaceuticals Srl, Milan, Italy
\end{abstract}

Purpose: Acute trauma pain management in the elderly population is a challenge. Inhaled methoxyflurane represents a promising treatment option; however, data in the elderly population are limited.

Patients and Methods: Subgroup, post hoc analysis including 69 patients aged $\geq 65$ years from a randomized, active-controlled, open-label study in the emergency setting. Key inclusion criterion was moderate-to-severe pain (Numerical Rating Scale [NRS] score $\geq 4$ ]) secondary to trauma in a single limb. Patients received inhaled methoxyflurane $(3 \mathrm{~mL})$ or standard analgesic treatment (SAT; IV paracetamol $1 \mathrm{~g}$ or ketoprofen $100 \mathrm{mg}$ for moderate pain [NRS $4-6]$ and IV morphine $0.1 \mathrm{mg} / \mathrm{kg}$ for severe pain [NRS $\geq 7]$ ). The primary endpoint was the overall change in visual analog scale (VAS) pain intensity from randomization to the next 3,5 , and $10 \mathrm{~min}$. Secondary endpoints included time to onset of pain relief (TOPR), efficacy up to 30 min, judgment of operators and patients, and safety.

Results: Pain reduction over time was similar in both groups. Median TOPR was shorter for methoxyflurane (9 min; 95\% CI: 7.8, $10.2 \mathrm{~min}$ ) than SAT (15 min; 95\% CI: 10.2, $19.8 \mathrm{~min}$ ). In terms of treatment satisfaction, patients and operators rated treatment efficacy and practicality, respectively, as "Excellent" or "Very good" 5.7 times and 3.4 times more frequently than SAT. A similar rate of adverse events (methoxyflurane: 6 events; SAT: 7 events) was recorded, all nonserious. No clinically significant changes in vital signs parameters were observed, and methoxyflurane did not result in cases of bradycardia or hypotension.

Conclusion: In elderly patients with trauma pain, inhaled methoxyflurane shows similar pain relief and safety compared to SAT, offering advantages in terms of onset of effect and user's satisfaction. Although this analysis presents some methodological limitations, it provides the first specific evidence of the use of inhaled methoxyflurane in the elderly population.

Trial Registration: EudraCT number: 2017-001565-25; Clinicaltrials.gov identifier NCT03585374.

Keywords: acute pain, trauma, elderly, analgesia, emergency department, methoxyflurane, prehospital

\section{Introduction}

Acute pain represents a critical and frequent issue in the emergency setting and requires an effective, safe, and manageable therapeutic approach. In elderly patients, treatment of acute pain presents additional difficulties due to cognitive and physical status, and concurrent conditions and medications. ${ }^{1}$ Commonly used
Correspondence: Alberto Farina Medical Affairs Department, Mundipharma Pharmaceuticals Srl, Milan, Italy

Tel +3902 318288I

Email alberto.farina@mundipharma.it 
drugs may cause potential adverse events (eg, nephrological and/or gastrointestinal events with nonsteroidal antiinflammatory drugs [NSAIDs] $)^{2}$ or may be inadequate for severe pain (eg, paracetamol). ${ }^{3}$ Opioids for severe pain in the elderly may cause cognitive impairment, delirium, respiratory depression, sedation, and other potentially severe side effects, ${ }^{4}$ limiting their routine use.

Low-dose-inhaled methoxyflurane (Penthrox ${ }^{\circledR}, 3 \mathrm{~mL}$ dose, Medical Developments International, Scoresby, Australia) is a non-opioid, volatile fluorinated analgesic, self-administered through an easy-to-use device handled by the patient. ${ }^{5-7}$ Methoxyflurane is currently available in Europe for the emergency relief of moderate-to-severe pain in conscious adults with trauma and associated pain, ${ }^{6}$ based on clinical evidence from a randomized, placebo-controlled trial (STOP!) conducted in UK emergency departments (EDs). ${ }^{8}$ More recently, the MEDITA (Methoxyflurane in Emergency Department in Italy) trial $^{9-11}$ demonstrated superiority of methoxyflurane versus standard analgesic treatment (SAT), consisting of intravenous (IV) paracetamol or ketoprofen for moderate pain and IV morphine for severe pain, in terms of pain reduction up to $30 \mathrm{~min}$ after randomization, onset of pain relief, and satisfaction of healthcare professionals and patients. Subgroup analyses according to baseline pain (moderate, severe) showed that methoxyflurane was more effective than SAT in both cases.

Currently, the evidence in the literature and the therapeutic indication refer to the whole adult population aged over 18 years, and specific efficacy and safety data in the elderly population are missing. The objective of this post-hoc analysis of the MEDITA study is to evaluate the efficacy, safety, and user-satisfaction of inhaled methoxyflurane vs SAT in patients with acute trauma pain aged 65 years or older.

\section{Patients and Methods}

\section{Study Design}

MEDITA was designed as a Phase IIIb, randomized, activecontrolled, parallel-group, open-label study. Patients were enrolled from 08 February 2018 to 22 February 2019 at 16 prehospital units and EDs in Italy. The study was registered with EudraCT number 2017-001565-25, and Clinicaltrials. gov identifier NCT03585374. Previous published papers reported methods and rationale of the protocol, ${ }^{9}$ results for the full study population, ${ }^{10}$ and results for the population with severe pain. ${ }^{11}$

Briefly, patients who went to the hospital emergency department on their own or were rescued in extra-hospital setting, and who had moderate (NRS 4-6) to severe (NRS 7-10) pain due to trauma in a single limb, were randomized (1:1) to inhaled methoxyflurane (experimental arm) or SAT (control arm). Treatment and evaluations were performed on the day of randomization, and 14 days after discharge, a phone call was made to gather additional information.

The study was performed following current and local ethical standards, Good Clinical Practices, 1964 Helsinki Declaration and its later amendments. Before the start of the study, Italian Medicines Agency (AIFA) and local Ethics Committees reviewed and approved the protocol. Each patient had to express their informed consent prior to enrollment. Due to clinical conditions, if the patient was unable to provide written consent, a verbal consent was obtained with the presence of an impartial witness, and the patient's signature was obtained as soon as conditions permitted. In addition to the monitoring carried out by the Contract Research Organization, an external GCP audit and a GCP inspection by AIFA have been carried out.

\section{Participants}

The original study included adult ( $\geq 18$ years old) patients with moderate-to-severe pain (numerical rating score $\geq 4$ ) secondary to a trauma (fracture, dislocation, crushing, contusion) to a single limb. In the present subgroup analysis, only patients aged $\geq 65$ years were included. This cut off was selected as it is commonly accepted to define an elderly population. The complete list of inclusion and exclusion criteria was previously reported by Fabbri et al. ${ }^{9}$ Briefly, patients were medically stable, conscious, and collaborating in order to express informed consent and follow the study procedures. Patients must not have taken any medicines for chronic pain or any other analgesic in the previous $5 \mathrm{~h}$ ( $8 \mathrm{~h}$ for diclofenac). Patients were excluded also in case of contraindications to methoxyflurane administration as per the Summary of Product Characteristics (SPC) ${ }^{12}$ or to any of the SAT.

\section{Randomization and Interventions}

Non-stratified blocks of four were used for randomization, and the procedure started with the verification of the eligibility criteria. Treatment allocation was concealed to investigators thanks to a centralized Interactive Web Response System integrated with the electronic case report form (eCRF). The study was open-label because of feasibility reasons: the sponsor and the investigator believed that adopting a double-blind, double-dummy design (to 
mask the different routes of administration) in the emergency context was too complex.

After randomization, drug administration took place in the shortest possible time. Patients in the experimental arm received only one $3 \mathrm{~mL}$ methoxyflurane inhaler, prepared by trained study staff in agreement with SPC recommendations. Administration was supervised by the investigator and started intermittently. Subsequently, patients could increase the frequency of inhalation or cover the diluting hole to obtain a greater analgesic effect, according to a patient-controlled analgesia. The patient's exhalation takes place through the same mouthpiece of the inhalation, in fact the outgoing air is filtered by activated carbon, preventing environmental release of methoxyflurane and potential occupational exposure. A single $3 \mathrm{~mL}$ inhaler provides approximately 25 to $60 \mathrm{~min}$ analgesia based on the intensity and frequency of inhalation of the individual patient. $^{12}$ The sponsor provided methoxyflurane in packs containing inhaler and a $3 \mathrm{~mL}$ methoxyflurane vial.

Patients randomized to the control arm received treatment according to the individual baseline pain intensity. Study procedures assigned patients with moderate pain (NRS 4-6) to IV paracetamol (1 g) or ketoprofen (100 mg), based on the practice of the individual center and any contraindications for the patient. Patients with severe pain (NRS $\geq 7$ ) were assigned to IV morphine $(0.10 \mathrm{mg} /$ $\mathrm{kg}$ ). Venous access, in agreement with local practice, was taken before randomization; then, dilution and infusion of the drug occurred within $10 \mathrm{~min}$. SATs were commercially available products supplied locally by study centers.

Rescue medications were up to the investigator's choice and were allowed at any time after randomization in case pain was not adequately controlled by study treatment.

\section{Study Assessments}

The primary objective of the full study was to demonstrate that the efficacy of inhaled methoxyflurane was noninferior to the SAT up to $10 \mathrm{~min}$, measured through the overall change in the VAS scale from baseline to 3, 5, and $10 \mathrm{~min}$. To collect additional efficacy data, pain intensity was measured up to $30 \mathrm{~min}(15,20,25$, and $30 \mathrm{~min})$, or until administration of rescue medication. Patients had to independently mark the VAS scale; however, if clinical conditions (eg, trauma to the hand) did not allow this operation, a properly trained operator could offer support. The NRS scale was used only for enrollment. For the efficacy measures, the VAS scale was chosen because it is continuous and able to increase the sensitivity and power of the study. Additional efficacy parameters included time from randomization to the onset of analgesic effect reported by the patient and administration of any rescue medication. The satisfaction parameters included the judgment of patients on efficacy and investigators on practicality. In both cases, the measurement was done with a 5-point Likert scale (poor, fair, good, very good, excellent) at $30 \mathrm{~min}$ after randomization.

Safety parameters were incidence of adverse events (AEs), serious AEs not related to the trauma presentation, and vital signs (systolic and diastolic blood pressure [SBP and DBP], heart rate, and respiratory rate). Safety evaluations were performed at baseline and 10 and 30 min after randomization.

For all evaluations, randomization was selected as baseline. Given that the next sequential operations were the preparation or dilution of the medicine, and therefore the start of administration, also the practicality and speed of preparation, in addition to the intrinsic effect of the drug, had a role on efficacy.

\section{Statistical Analysis}

To compare the VAS pain intensity variation from baseline between study treatments, a linear mixed-effect model for repeated measures adjusted for VAS baseline score, and the interaction between time point and treatment was used. The primary analysis was the overall test for treatment effect at 3,5 , and $10 \mathrm{~min}$. For each analysis, the treatment difference (methoxyflurane-control) was presented with 95\% confidence interval (CI): if the upper limit was $<1$, noninferiority of methoxyflurane vs control was concluded; if the upper limit was $<0$, superiority was concluded.

The mean change from baseline in VAS pain intensity at following time points $(15,20,25,30 \mathrm{~min})$ was estimated for each group with $95 \% \mathrm{CI}$ and the $t$-test for equality of means was used to compare treatment effects. Time to onset of pain relief was presented with Kaplan-Meier curves, with "survival time" representing the event "onset of pain relief". Frequency of rescue medication administration between groups was tested using Fisher's exact test. User satisfaction (efficacy for patients and practicality for investigators) between groups was compared using the Mann-Whitney $U$-test. For efficacy variables, the intention-to-treat (ITT) population approach was adopted, and no imputation of missing data was performed.

Only analyses of the ITT population were performed since it was a post-hoc, exploratory study on a small 
population, and since in the total population ${ }^{10}$ the ITT and PP results were similar. Furthermore, only 2 cases would have been excluded in the PP analysis.

AEs were classified using the Medical Dictionary for Regulatory Activities version 22.0 and presented for the safety population (based on administered treatment). Statistics were performed with IBM SPSS Statistics version 23.0.

\section{Sample Size}

The sample size calculation for the full study is reported in a previous paper ${ }^{9}$ and planned to randomize 136 patients per treatment arm. In this report, data from 69 elderly patients are presented, and as a post-hoc analysis, a formal sample size was not calculated. Thus, the present analysis was conducted in an exploratory manner only. Furthermore, the proportion of elderly patients at the beginning of the study was not predictable, thus.

\section{Results}

\section{Study Patients}

Among the 272 patients randomized in the full study, ${ }^{10} 69$ (25\%) were $\geq 65$ years of age and included in the present post hoc analysis. Baseline characteristics of elderly patients subgroup are shown in Table 1; 35 (50.7\%, mean age 75) of those received methoxyflurane and 34 (49.3\%, mean age 76.4$)$ received SAT. In both groups, most patients were female and Caucasian, a similar proportion of severe and moderate pain level was present. Contusion and fracture were the most common suspected injury types at inclusion. In the control group, all patients with severe pain $(15 ; 44.1 \%)$ were treated with IV morphine, and patients with moderate pain were treated with IV paracetamol $(16 ; 47.1 \%)$ or IV ketoprofen $(3 ; 8.8 \%)$. At baseline, mean VAS pain intensity was $73( \pm 18[\mathrm{SD}])$ in the overall elderly population, $71( \pm 20[\mathrm{SD}])$ in the methoxyflurane group, and $76( \pm 16[\mathrm{SD}])$ in the control group.

\section{Efficacy Results}

Pain reduction according to VAS over time was very similar in both study groups (Figure 1). Median time to onset of pain relief was shorter for methoxyflurane ( $9 \mathrm{~min}$; 95\% CI: $7.8,10.2 \mathrm{~min})$ than SAT (15 min; $95 \%$ CI: 10.2 , $19.8 \mathrm{~min}$ ), with the faster time to pain relief for methoxyflurane evident in Kaplan-Meier curves up to $\sim 25 \mathrm{~min}$ (Figure 2). At $10 \mathrm{~min}$ about $80 \%$ of patients reported pain relief the methoxyflurane group vs about $40 \%$ of
Table I Baseline Characteristics

\begin{tabular}{|c|c|c|c|}
\hline Characteristics & & $\begin{array}{l}\text { Methoxyflurane } \\
(\mathrm{N}=35)\end{array}$ & $\begin{array}{l}\text { Standard } \\
\text { Analgesic } \\
\text { Treatment (SAT) } \\
(\mathrm{N}=34)\end{array}$ \\
\hline Age (years) & $\begin{array}{l}\text { Mean (SD) } \\
\text { Range }\end{array}$ & $\begin{array}{l}75(6.8) \\
65-91\end{array}$ & $\begin{array}{l}76,4(7.7) \\
65-95\end{array}$ \\
\hline Gender [n (\%)] & $\begin{array}{l}\text { Male } \\
\text { Female }\end{array}$ & $\begin{array}{l}5(14) \\
30(86)\end{array}$ & $\begin{array}{l}6(18) \\
28(82)\end{array}$ \\
\hline Weight (kg) & $\begin{array}{l}\text { Mean (SD) } \\
\text { Range }\end{array}$ & $\begin{array}{l}69(10.2) \\
50-94\end{array}$ & $\begin{array}{l}69(10.3) \\
50-85\end{array}$ \\
\hline Race [n (\%)] & $\begin{array}{l}\text { Caucasian } \\
\text { Asian } \\
\text { Black } \\
\text { Other }\end{array}$ & $\begin{array}{l}34(97) \\
1(3.0) \\
0 \\
0\end{array}$ & $\begin{array}{l}34(100) \\
0 \\
0 \\
0\end{array}$ \\
\hline $\begin{array}{l}\text { Baseline pain } \\
\text { group [n (\%)] }\end{array}$ & $\begin{array}{l}\text { Moderate } \\
{[\text { NRS 4-6] }} \\
\text { Severe } \\
{[N R S \geq 7]}\end{array}$ & $\begin{array}{l}18(51) \\
17(49)\end{array}$ & $\begin{array}{l}19(56) \\
15(44)\end{array}$ \\
\hline $\begin{array}{l}\text { Suspected injury } \\
\text { type at inclusion } \\
\text { [n (\%)] }\end{array}$ & $\begin{array}{l}\text { Contusion } \\
\text { Fracture } \\
\text { Dislocation } \\
\text { Crushing }\end{array}$ & $\begin{array}{l}15(43) \\
15(43) \\
5(14) \\
0\end{array}$ & $\begin{array}{l}15(44) \\
13(38) \\
5(15) \\
1(3)\end{array}$ \\
\hline
\end{tabular}

patients in the SAT group. There was no difference between the treatment groups in the proportion of patients who received rescue analgesic medication (methoxyflurane: 2 patients [5.7\%]; SAT: 1 patient [2.9\%]; $\mathrm{p}=1.000$ ).

In terms of treatment satisfaction, patients rated treatment efficacy as "Excellent" or "Very good" 5.7 times more frequently than SAT (34.3\% vs $6 \%$ ), and healthcare professionals rated practicality as "Excellent" or "Very good" 3.4 times more frequently than SAT ( $60.0 \%$ vs $17.7 \%)$. Overall, the distribution of patients' efficacy ratings and physicians' practicality was significantly different between treatments $(p=0.040$ and $p=0.003$, respectively, Figure 3$)$.

\section{Safety Results}

A total of 13 adverse events (methoxyflurane: 6 events; SAT: 7 events) were recorded during the study (all nonserious; Table 2). Six events were related or possibly related to study treatment (methoxyflurane, 4 events: 1 vertigo, 2 nausea, 1 somnolence; paracetamol, 2 events: 1 presyncope, 1 hypotension). Three patients discontinued methoxyflurane treatment due to AEs.

There were no clinically significant changes in vital signs parameters (blood pressure, heart rate, respiratory rate) in both groups, complete data are reported in Tables S1-S9. 


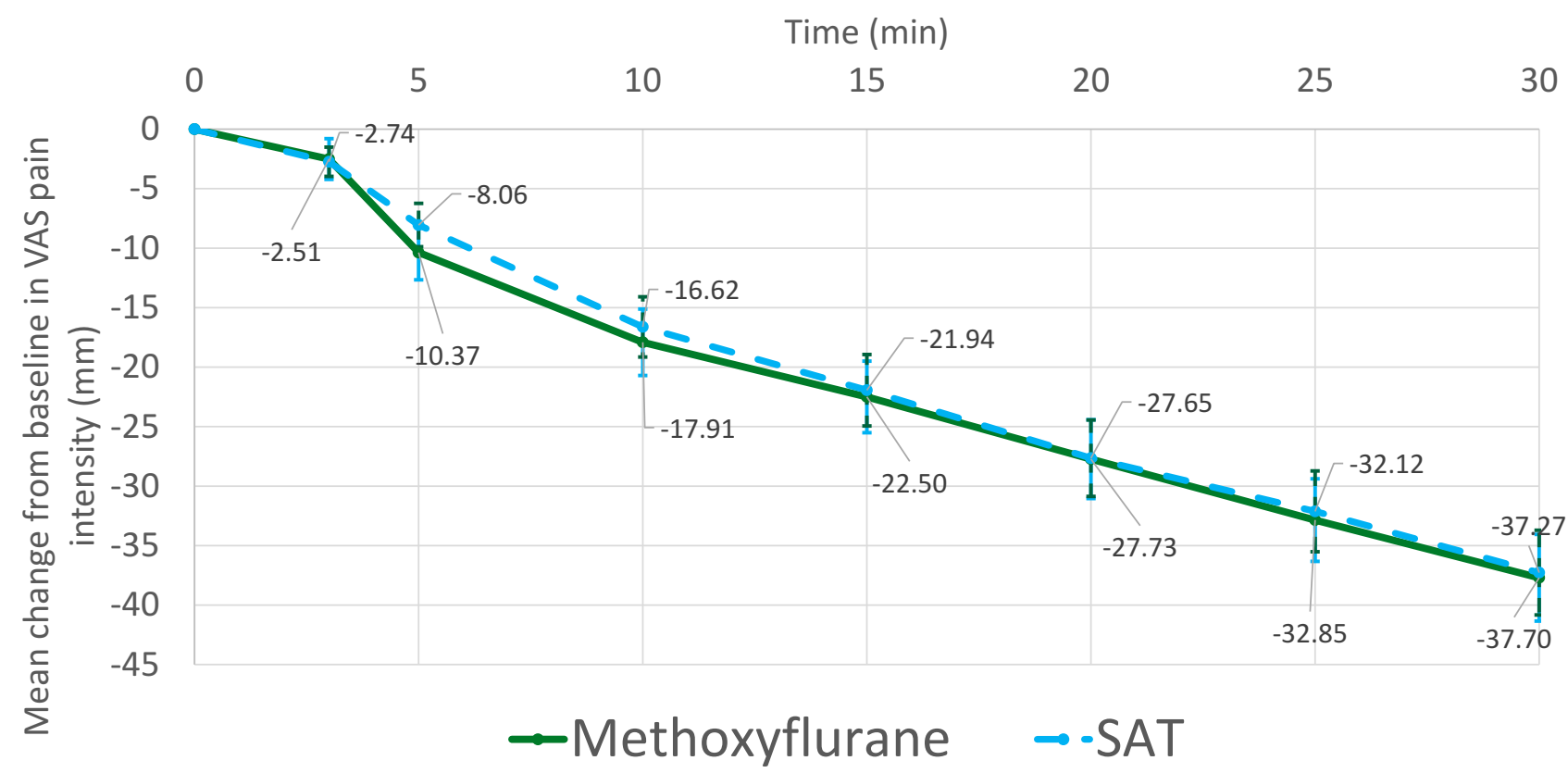

Figure I Pain reduction. Mean \pm standard error. $\mathrm{n}=69$. SAT: standard analgesic treatment (moderate pain: IV paracetamol or IV ketoprofen; severe pain: IV morphine).

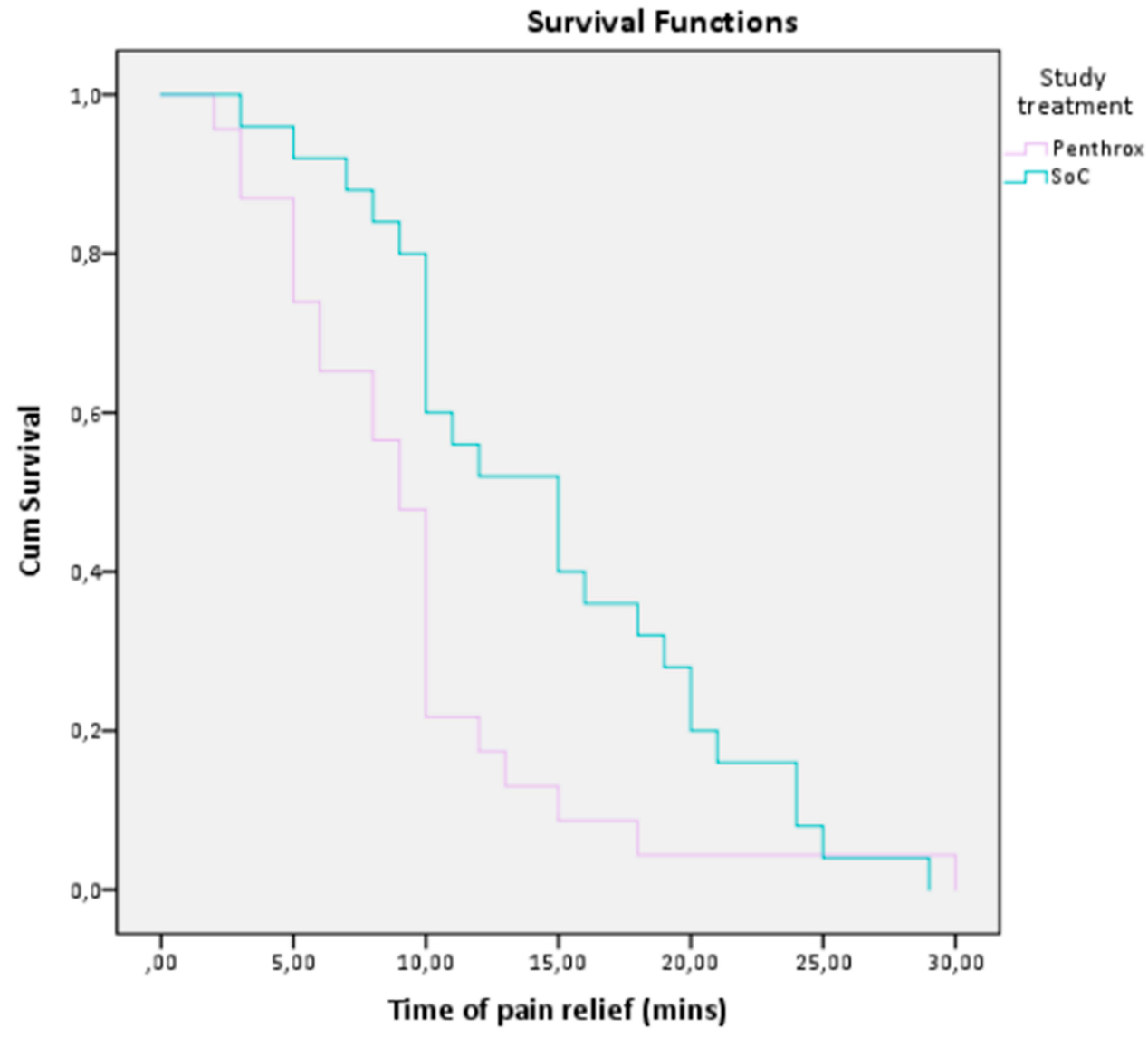

Figure 2 Time to pain relief. 


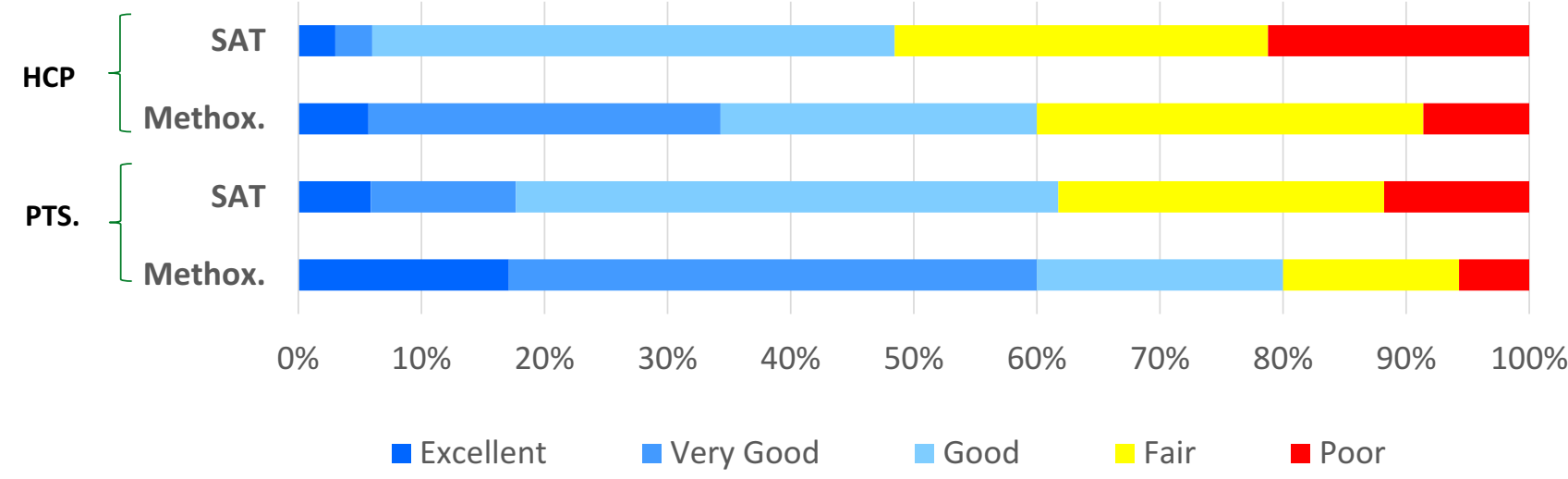

Figure 3 Overall treatment efficacy evaluated by patients and practicality by HCPs. Cumulative proportions of 5-point Likert qualitative scale ("Poor”, "Fair”, "Good”, “Very Good", or "Excellent") at 30 min after randomization. SAT: standard analgesic treatment (moderate pain: IV paracetamol or IV ketoprofen; severe pain: IV morphine). P-value $=0.04$ (Mann-Whitney U-test).

\section{Discussion}

This subgroup analysis of the randomized, activecontrolled trial MEDITA showed similar efficacy and safety of inhaled methoxyflurane compared to SAT in terms of pain intensity up to $30 \mathrm{~min}$ after randomization. On the other hand, inhaled methoxyflurane showed two relevant advantages over SAT: faster onset of analgesia, and higher user satisfaction. To the best of our knowledge, this is the first study which specifically addresses the use of inhaled methoxyflurane in the elderly population. Our results support the use of the inhaled methoxyflurane in the elderly population as a new therapeutic option, able to offer a faster reduction of pain and a better user experience. Given the urgency and complexity of pain management in the emergency situations, and the peculiarity of the study population, these results are clinically relevant.

As recommended by current SmPC, ${ }^{12}$ caution should be paid in elderly patients because of potential effects on blood pressure and heart rate were reported with high dose methoxyflurane used in anesthesia. As the risk may potentially be increased for older people with hypotension and bradycardia, caution should be paid in the elderly due to possible reduction in blood pressure. According to the exclusion criteria of the present study, patients had normal signs at inclusion. Overall, a low number of adverse events, all non-serious and transient, occurred in the study, and vital signs did not change significantly. Since analgesia is controlled by the patient, it is possible to control and limit the occurrence of adverse events related to drug exposure, modulating drug intake according to subjective need and tolerability. Furthermore, since the overall dose is low (up to $6 \mathrm{~mL}$ with 2 consecutive administrations allowed in clinical practice), the risk of overdose is reduced. Any adverse event can be controlled through the temporary or permanent suspension of treatment. Therefore, our results support a safe use of inhaled methoxyflurane in the elderly population. For these patients, an additional therapeutic option may be useful, since comorbidities and potential adverse or contraindication may limit the use of other common agents, eg, NSAID, and opioids. In addition, intravenous drugs may be non-administrable due to the low venous patrimony in some patients.

Table 2 Adverse Events

\begin{tabular}{|c|c|c|c|c|c|c|}
\hline Adverse Events & Paracetamol $(\mathrm{N}=16)$ & Paracetamol - \% & Methoxyflurane $(\mathrm{N}=35)-\mathrm{N}$ & Methoxyflurane - \% & Total $(\mathrm{N}=69)-\mathrm{N}$ & Total - \% \\
\hline Vertigo & 0 & $0.0 \%$ & I & $2.9 \%$ & I & $1.4 \%$ \\
\hline Constipation & I & $6.3 \%$ & 0 & $0.0 \%$ & I & $1.4 \%$ \\
\hline Nausea & I & $6.3 \%$ & 2 & $5.7 \%$ & 3 & $4.3 \%$ \\
\hline Vomiting & 1 & $6.3 \%$ & 0 & $0.0 \%$ & 1 & $1.4 \%$ \\
\hline Bronchitis & 0 & $0.0 \%$ & I & $2.9 \%$ & I & $1.4 \%$ \\
\hline Presyncope & 2 & $12.5 \%$ & 1 & $2.9 \%$ & 3 & $4.3 \%$ \\
\hline Somnolence & 0 & $0.0 \%$ & I & $2.9 \%$ & I & $1.4 \%$ \\
\hline Hyperhidrosis & I & $6.3 \%$ & 0 & $0.0 \%$ & 1 & $1.4 \%$ \\
\hline Hypotension & 1 & $6.3 \%$ & 0 & $0.0 \%$ & 1 & $1.4 \%$ \\
\hline
\end{tabular}


The higher user's satisfaction is a particularly relevant result in this setting. The elderly population often presents complex and fragile conditions and is generally more suspicious of new technologies than young people. Inhaled methoxyflurane offers less invasive treatment than IV therapies, and requires active patient participation in controlling analgesia. In an emergency context, both in the hospital and especially in an out-of-hospital setting, a better perception of the therapy may potentially reduce tension/stress and facilitate the treatment process.

Treatment of acute pain is important to stop the stress reaction. The stress reaction triggered by pain causes physiopathological reactions such as increased arterial pressure, tachycardia, tachypnea, and other endocrine-metabolic phenomena ${ }^{13}$ which can precipitate the clinical pictures in patients with comorbidities and reduced functional reserves such as the elderly. Follow-up studies on long-term posttraumatic pain have shown that pain at the time of trauma is considered the main independent factor of chronic and longterm pain. ${ }^{14}$ In this perspective, it is important to relieve acute trauma pain quickly and effectively.

The limitations of the present study include the exploratory, post-hoc nature of the analysis, and the absence of sample size calculation to detect differences of efficacy and safety between the two groups. For this reason, the results, although encouraging and in line with the previous evidence in the literature, must be interpreted with caution and cannot be considered definitive. Additional limitations of the study, as previously discussed, ${ }^{10}$ include the open-label design which represents an intrinsic bias, but it was necessary to guarantee the feasibility of the study considering the emergency setting. Given these limitations, further adequately designed head-to-head studies are needed to detect treatment differences in the efficacy of inhaled methoxyflurane vs SAT in elderly patients with trauma pain.

In conclusion, inhaled methoxyflurane showed similar pain relief and safety compared to IV standard in elderly patients with moderate to severe trauma pain, providing advantages in terms of onset of effect and user's satisfaction. Although the study presents a number of limitations due to the post hoc analysis of a randomized open-label study, it provides the first specific evidence of the use of inhaled methoxyflurane in the elderly population.

\section{Data Sharing Statement}

The datasets generated, analyzed and reported within this manuscript may be requested in accordance with the Data
Sharing Policy of Mundipharma Research Limited available from www.mundipharma-rd.eu.

\section{Ethics Approval and Informed Consent}

The trial was approved by the Italian Medicines Agency (AIFA). The co-ordinating ethics committee, Comitato Etico Regione Toscana - Area Vasta Centro, Florence, Italy, approved the trial protocol on 1 December 2017. The trial was approved by local Ethics Committees at each center. Written informed consent was obtained from all patients before enrollment. Registration in public databases: EudraCT (2017-001565-25), 02 March 2018; ClinicalTrials.gov (NCT03585374), 13 July 2018.

\section{Acknowledgments}

The investigators of the MEDITA Study Group are: Germana Ruggiano, Guido Tota, Michela Zerini, Alessandra Semino, Sara Rasla, Elena Mecatti, Gabriele Panci, Catia Fronduti, Patrizia Signoroni, Alessandro Corradini, Alessandra Benucci (Emergency Medicine Department, Santa Maria Annunziata Hospital, Florence, Italy); Isabella Bartoli, Davide Messina (SUES 118 Catania-Ragusa-Siracusa, Cannizzaro Hospital, Catania, Italy); Giuseppe Carpinteri, Paola Noto, Chiara Giraffa (Department of Emergency Medicine, Vittorio Emanuele University Hospital, Catania, Italy); Andrea Fabbri (Department of Emergency Medicine, Morgagni-Pierantoni Hospital, Forlì, Italy); Francesco Bermano (118 Service, San Martino Hospital, Genova. Italy); Maurizio Chiesa; Beatrice Sabini (Emergency Department, S. Antonio Hospital - ULSS 16, Padova, Italy); Mario Oppes, Vincenzo Pretti (Emergency Department, Sassari University Hospital, Sassari, Italy); Peppino Masciari, Antonietta Comito, Lucia Orlando (Emergency Department, Pugliese-Ciaccio Hospital, Catanzaro, Italy); Davide Torti, Giorgio Carbone, Gianfrancesco Alberto (Emergency Department, Gradenigo Hospital, Torino, Italy); Vittorio Iorno (Anesthesia, Resuscitation and Emergency Department, Fondazione IRCCS Cà Granda Ospedale Maggiore Policlinico di Milano, Milan, Italy); Antonio Voza, Alessandro Barraco, Fabio Intelligente, Monica Suardi, Claudio Addari (Emergency Department, IRCCS Humanitas Research Teaching Hospital, Rozzano, Milan, Italy); Piero Paolini, Nicola Bertocci (118 Unit, Azienda USL Toscana Centro, Pistoia, Italy); Sossio Serra, Stefano Tranelli, Pasquale Di Conza, Alessandro Valentino, Patrizia Cuppini, Elisabetta Lucchi, Carlo Garaffoni (Emergency Department, 
Maurizio Bufalini Hospital, Cesena, Italy); Gianfilippo Gangitano, Giuseppe D’Antuono, Lorena Bertozzi, Giulia Montanari (Emergency Department, Infermi Hospital, Rimini, Italy), Raffaella Francesconi (Faenza Hospital, Faenza, Italy).

We thank the Contract Research Organization YGHEA, Division of Ecol Studio SPA, Bologna, for the operational support.

Penthrox $^{\circledR}$ is a registered trade mark of Medical Developments International (MDI) Limited and used under license.

\section{Author Contributions}

All named authors meet the International Committee of Medical Journal Editors (ICMJE) criteria for authorship for this manuscript, take responsibility for the integrity of the work as a whole, and have given final approval to the version to be published.

All authors contributed to data analysis, drafting or revising the article, gave final approval of the version to be published, and agree to be accountable for all aspects of the work.

\section{Funding}

The study was sponsored and funded by Mundipharma Pharmaceuticals srl, Milan, Italy. The study sponsor was involved in study design and overview, data interpretation, and report revision. The sponsor had no role in data collection or analysis. The corresponding author had full access to all the data in the study and had final responsibility for the decision to submit for publication. Editorial costs were funded by Mundipharma Pharmaceuticals srl.

\section{Disclosure}

Antonio Voza, Sossio Serra, Germana Ruggiano reports nonfinancial support from Mundipharma, Andrea Fabbri, have nothing to disclose. Elisabetta Bonafede is an employee of the clinical research organization that conducted the study. Antonella Sblendido, Amedeo Soldi and Alberto Farina are employees of Mundipharma Pharmaceuticals srl.

Journal of Pain Research

\section{Publish your work in this journal}

The Journal of Pain Research is an international, peer reviewed, open access, online journal that welcomes laboratory and clinical findings in the fields of pain research and the prevention and management of pain. Original research, reviews, symposium reports, hypothesis formation and commentaries are all considered for publication. The manuscript

Submit your manuscript here: https://www.dovepress.com/journal-of-pain-research-journa

\section{References}

1. Kaye AD, Baluch A, Scott JT. Pain management in the elderly population: a review. Ochsner J. 2010;10(3):179-187.

2. Boers M, Tangelder MJ, van Ingen H, Fort JG, Goldstein JL. The rate of NSAID-induced endoscopic ulcers increases linearly but not exponentially with age: a pooled analysis of 12 randomised trials. Ann Rheum Dis. 2007;66(3):417-418. doi:10.1136/ard.2006.055012

3. Raccomandazioni Intersocietarie Italiane (SIAARTI, SIMEU, SIS 118, AISD, SIARED, SICUT, IRC) sulla gestione del dolore in emergenza. Available from: http://www.aisd.it/e107_files/downloads/raccintersocie tarie_it_complete31052014.pdf. Accessed February 27, 2020.

4. Chau DL, Walker V, Pai L, Cho LM. Opiates and elderly: use and side effects. Clin Interv Aging. 2008;3(2):273-278. doi:10.2147/cia. s1847

5. Hansen MS, Dahl JB. Limited evidence for intranasal fentanyl in the emergency department and the prehospital setting: a systematic review. Dan Med J. 2013;60:A4563.

6. Porter KM, Dayan AD, Dickerson S, Middleton PM. The role of inhaled methoxyflurane in acute pain management. Open Access Emerg Med. 2018;10:149-164. doi:10.2147/OAEM.S181222

7. Jephcott C, Grummet J, Nguyen N, Spruyt O. A review of the safety and efficacy of inhaled methoxyflurane as an analgesic for outpatient procedures. Br J Anaesth. 2018;120:1040-1048. doi:10.1016/j. bja.2018.01.011

8. Coffey F, Wright J, Hartshorn S, et al. STOP!: a randomised, double-blind, placebo-controlled study of the efficacy and safety of methoxyflurane for the treatment of acute pain. Emerg Med J. 2014;31:613-618. doi:10.1136/emermed-2013-202909

9. Fabbri A, Carpinteri G, Ruggiano G, et al. Methoxyflurane versus standard of care for acute trauma-related pain in the emergency setting: protocol for a randomised, controlled study in Italy (MEDITA). Adv Ther. 2019;36:244-256. doi:10.1007/s12325-0180830-x

10. Mercadante S, Voza A, Serra S, et al.; MEDITA Study Group. Analgesic efficacy, practicality and safety of inhaled methoxyflurane versus standard analgesic treatment for acute trauma pain in the emergency setting: a randomised, open-label, active-controlled, multicentre trial in Italy (MEDITA). Adv Ther. 36;2019:3030-3046. doi:10.1007/s12325-019-01055-9

11. Voza A, Ruggiano G, Serra S. Inhaled methoxyflurane versus intravenous morphine for severe trauma pain in the emergency setting: subgroup analysis of MEDITA, a multicenter, randomized, controlled, open-label trial. J Pain Res. 2020;13:1-12. doi:10.2147/ JPR.S240911

12. Penthrox ${ }^{\circledR}$ summary of product characteristics. Available from: https:// www.medicines.org.uk/emc/medicine/31391. Accessed November 27, 2019.

13. Desborough JP. The stress response to trauma and surgery. $\mathrm{Br}$ J Anaesth. 2000;85(1):109-117. doi:10.1093/bja/85.1.109

14. Castillo RC, MacKenzie EJ, Wegener ST, Bosse MJ; LEAP Study Group. Prevalence of chronic pain seven years following limb threatening lower extremity trauma. Pain. 2006;124(3):321-329. doi:10.1016/j.pain.2006.04.020

management system is completely online and includes a very quick and fair peer-review system, which is all easy to use. Visit http:// www.dovepress.com/testimonials.php to read real quotes from published authors. 